SEMICONDUCTORS Nothing is the Last Word

from a Correspondent

Those were the words of Professor C. A. Coulson (University of Oxford) registering surprise that his description of the vacancy in diamond, developed eleven years ago with Mary Kearsley, should have survived so long. He was making an all-too-brief appearance for the panel discussion on theoretical aspects of defects at the International Conference on Defects in Semiconductors held in Reading from July 19 to 21. Dr A. B. Lidiard (AERE, Harwell) was chairman for the panel discussion, and other participants included Dr G. D. Watkins and Dr R. Messmer (General Electric Company, USA) who are currently enjoying much success in treating defects in semiconductors using Extended Hückel Theory (EHT), a theoretical technique taken over from chemistry. Debate centred on the provision in EHT of only one electron states, that is the strict localization of electrons. Drs Watkins and Messmer were the first to recognize this drawback, and they suggested that where the states fell within the usual bands of allowed energy state delocalization would occur. Professor Coulson thought this in the best chemical tradition.

The conference was the sixth in a series started at Gatlinburg in 1958 when some of the first work at liquid helium temperatures was reported. No satisfactory explanation of that work has yet been advanced, but hopes were raised at Reading that such an explanation might soon be forthcoming. Besides such continuing lines of enquiry, the field has since embraced work on ion implantation and amorphous materials although the organizers were careful only to include contributions in these areas which were strictly linked to a study of radiation damage.

Professor J. W. Corbett (State University of New York) gave the first invited paper on defect production and defect production processes. Having cooperated very closely with Drs Watkins and Messmer, he discussed the possibility of very low energy defect migration and production processes, which appear to follow from the "bond centred" and "split" interstitial configurations, now suggested by EHT, together with the effects of charge state on their stability. It was here that some hope of explaining the work at low temperatures appeared.

Apart from their contributions to the panel discussion Dr A. B. Lidiard and Dr G. D. Watkins gave reviews on defect energies and states, and on vacancies and interstitials in semiconductors, respectively. A review by Dr R. C. Newman (University of Reading) and $\mathrm{Dr}$
R. Peart on the role of defects in diffusion produced the general prescription that most existing work should be looked at again, and closely. Results in ion implantation were discussed by $\mathrm{Dr}$ F. L. Vook (Sandia Laboratories, USA) with special reference to the way in which it can elucidate known defect centres, for instance by isotopic substitution. Work on devices was reviewed by Dr B. L. Gregory (Sandia Laboratories) but unfortunately there were no contributions. Dr R. S. Nelson (AERE, Harwell) presented observations on highly disordered materials using the electron microscope, and the potentialities of helium ion channelling techniques for evaluating disorder were clearly set out by Dr W. L. Brown (Bell Laboratories, USA).

\title{
More Evidence for Repressed Messengers
}

WAY back in 1963, McAuslan noticed that actinomycin $\mathrm{D}$ could prolong by many hours the accumulation of virusspecific thymidine kinase in pox-infected HeLa cells. He proposed that during the normal course of infection an RNA was made between 2 and 4 hours, which repressed the expression of the mRNA for TK made before that time.

Soon afterwards, the idea of posttranscriptional repression of preformed mRNA function was taken up again by Gordon Tomkins, also to explain paradoxical effects of actinomycin $\mathrm{D}$ on enzyme induction. Hepatoma tissue culture (HTC) cells exposed to adrenal corticosteroids show an increase by a factor of ten to fifteen in the amount and rate of synthesis of tyrosine aminotransferase (TAT), which reaches a steady state with enzyme degradation in about 12 hours. If the steroid is removed at this time there is, within 6 hours, a decline in the amount of enzyme and its rate of synthesis to the basal level. If, however, $5 \mu \mathrm{g} / \mathrm{ml}$. of actinomycin $\mathrm{D}$ is given when the steroid is removed, the enzyme level increases ("superinduction") or remains at the same level for many hours. From these and many other observations, Tomkins and his colleagues have devised a model for the control of TAT synthesis which involves two genes-one for the mRNA of TAT itself, and another for a labile RNA or protein repressor molecule which binds to TAT mRNA and both prevents its translation and promotes its degradation (Tomkins et al., Science, 166, 1474; 1969). According to this model steroids could act entirely at the transcriptional level by promoting the synthesis of TAT mRNA and shutting off the synthesis of the labile repressor RNA, or they could work post-transcriptionally by inactivating the repressor and allowing the TAT mRNA to accumulate (provided that it was already being synthesized continuously). High levels of actinomycin $D$ are thought to inhibit the synthesis of the labile repressor, thereby releasing any inactive mRNA-repressor complexes and increasing the rate of synthesis of TAT ("messenger rescue").

Although this model, in one form or another, has been around for a long time, consideration of it has been, in
Tomkins's own words, "rather restrained". In part this has resulted from confusion over the interpretation of the "superinduction" by actinomycin D of TAT activity. Kenney and his collaborators, working with a different hepatoma cell line (H35), under conditions in which TAT was induced in monolayers maintained in Eagle's basal medium without serum, found that enzyme turnover after removal of the steroid was strongly inhibited by high doses of actinomycin D. They therefore argued that "superinduction" was an artefact produced by this inhibition of TAT degradation. Tomkins's reply to this was to show that under these poor nutritional, stepdown, conditions, the degradation of TAT was faster than in an enriched medium, and that while such "enhanced" degradation was indeed inhibited by actinomycin $\mathrm{D}$, turnover under the conditions normally used in his laboratory was not affected by the antibiotic. Instead, he has consistently argued that "superinduction" is a result of an increased rate of synthesis of TAT, as predicted by the post-transcriptional control model, and in an article in Nature New Biology next week (September 6) he presents new evidence in favour of this interpretation.

Obviously, until direct evidence for translational repression of TAT messenger function is available, the model must remain hypothetical and open to alternative explanations, however unlikely. (For example, in the presence of high doses of actinomycin $\mathrm{D}$, labile mRNAs will be lost and such things as initiation factors, which may be limiting for protein synthesis, may become available for increased translation of the more stable mRNAs.) Meanwhile, in his article in Nature New Biology, Tomkins presents an impressive list of proteins and enzymes like the pox-virus thymidine kinase cited earlier, the concentrations of which have been reported to be stimulated by actinomycin $\mathrm{D}$ or other inhibitors of RNA synthesis. Some of these effects may, of course, result from an inhibition of protein degradation, but others, it is argued, may turn out to reveal a widespread use of post-transcriptional regularity elements in the control of the utilization of mRNA in animal cells. 